\title{
Evaluation Of Thyroid Function Tests In Patients With Acute Kidney Injury
}

\author{
Hanişe Ozkan1, Yildiz Okuturlar², Hakan Kocoglu², Irem Kiraç Utku', Esra Demir ${ }^{1}$, Ozlem Harmankaya ${ }^{2}$, Mehmet Palaz², Bugra \\ Oztosun², Meral Mert ${ }^{3}$, Aysegul Kudu', Sibel Kocak Yucel ${ }^{4}$, Abdulbaki Kumbasar ${ }^{2}$ \\ 1- Kanuni Sultan Suleyman Education and Research Hospital, Department Of Internal Medicine, Istanbul \\ 2- Bakırkoy Dr. Sadi Konuk Education and Research Hospital, Department Of Internal Medicine, Istanbul \\ 3- Bakırkoy Dr. Sadi Konuk Education and Research Hospital, Department Of Endocrinology, Istanbul \\ 4- Bakırkoy Dr. Sadi Konuk Education and Research Hospital, Department Of Nephrology, Istanbul
}

Aim: Euthyroid sick syndrome can be described as abnormal findings on thyroid function tests that occur in the setting of a nonthyroidal illness (NTI) without preexisting hypothalamic-pituitary and thyroid gland dysfunction. In this study we tried to compare thyroid function tests between patients with acute kidney injury (AKI) and healthy controls.

Materials and Methods: This study consisted of 31 patients with AKI and 26 healthy controls. All patients' physical examination findings, laboratory data, comorbid illnesses were recorded and compared with healthy subjects.

Results: Of the patients group $69.2 \%$ patients were female and of the control group $64.5 \%$ patients were female. Also of the patients $25.8 \%$ had diabetes mellitus, $16.1 \%$ had hypertension, $\% 16.1$ had cancer, $12.9 \%$ had cardiovascular disease, $9.7 \%$ had acute gastroenteritis, $38.7 \%$ had urinary tract infection, $9.7 \%$ had pneumonia. Mean creatinine value in the patients group was $4.70 \pm 2.17$ and in the control group was $1.10 \pm 0.28 \mathrm{mg} / \mathrm{dL}$ $(p=0.0001)$. There were no significant differences in TSH and fT4 levels between patients and controls whereas fT3 levels were significantly lower in patients group ( $p=0.0001)$. No correlation was determined between creatinine and age, body mass index, fT4, TSH but there was a positive correlation between creatinine and urea $(r=0.691, p=0.0001)$ and there was a negative correlation between creatinine and fT3 ( $r=-0.691, p=0.0001)$. Discussion: The frequency of thyroid function abnormalities is related to the magnitude of the illness. The most common abnormality is a T3 reduction, occurring in about $40-100 \%$ of cases of $\mathrm{NTI}$, which parallels the increase of rT3. As the disease severity increases, T4 levels also decrease. Most patients who are critically ill have reduced T4 levels. In patients who are hospitalized with an $\mathrm{NTI}$, about $10 \%$ have abnormally low TSH values. As in chronic kidney disease, fT3 levels could be a marker for euthyroid sick syndrome in patients with AKI. 